\title{
Self-healing performance of water triggered smart coating characterized by local electrochemical techniques
}

\author{
Wei Wang ${ }^{1,2, a^{*}}$, Likun Xu ${ }^{1, b}$ and Xiangbo Li $^{1, c}$ \\ ${ }^{1}$ State Key Laboratory for Marine Corrosion and Protection, Luoyang Ship Material Research \\ Institute(LSMRI). 149-1 Zhuzhou Road, Laoshan District, Qingdao, 266100, PR China. \\ ${ }^{2}$ Key Laboratory of Marine Environmental Corrosion and Bio-fouling, Institute of Oceanology, \\ Chinese Academy of Sciences. 7 Nanhai Road, Qingdao, 266101, PR China.
}

awwei020404@163.com, ${ }^{\mathrm{b}}$ xulk@sunrui.net, ${ }^{\mathrm{c}}$ Lixb@ sunrui.net

Keywords: Self-healing, Microcapsules, Smart Coating, Anticorrosion.

\begin{abstract}
Scratched alkyd varnish coating (AVC) with isophorone diisocyanate (IPDI) microcapsules as functional additives display self-healing properties. Here, we investigated the mechanism of the self-healing process using a water trigger in seawater. FT-IR spectra of the self-healing products in the scratched crevice confirmed that the scratched crevice was healed by self-healing product. Scanning micro-reference electrode (SMRE) and alternating current scanning electrochemical microscopy were used to monitor the self-healing process of IPDI-AVC. This study enhances the comprehension of the self-healing mechanism of self-healing AVC-coatings functionalized by the addition of microcapsules.
\end{abstract}

\section{Introduction}

Corrosion process always occurs in reinforcing steel of the buildings and metal architectures. It is estimated that corrosion destroys one quarter of the world's annual steel production, which corresponds to about 150 million tons per year, or 5 tons per second [1]. Therefore, it is necessary to inhibit the corrosion process of reinforcing steel. The coating is one of the most effective methods to protect the reinforcing steel. However, the conventional coating repair methods are not effective for healing invisible micro-cracks within the structure during its service life. In response, the concept of self-healing polymeric materials was proposed in the 1980s [2] as a means of healing invisible micro-cracks for extending the working life and safety of the polymeric components. The more recent publications in the topic by White et al. [3] in 2001 further inspired world wide interests in these materials[4]. Corrosion process of reinforcing steel always coupled by water, as water is the carrier of materials, electrons and reaction place. Thus, corrosion could be inhibited when the self-healing process inhibits the water transportation. Isocyanates are reactive with water and are thus potential catalyst-free healing agents for self-healing materials that are exposed to moist or wet environments. Yang et al.[5] first encapsulated isophorone diisocyanate (IPDI) as a healing agent via the interfacial polymerization reaction of Toluene diisocyanate prepolymer and 1,4-butanediol in an O/W emulsion. Wang et al.[6-8] then reported the microencapsulation of liquid isocyanate monomer. Hexamethylene diisocyanate was encapsulated by polyurethane microcapsules based on the polymerization of methylene diphenyl diisocyanate prepolymer and 1,4-butanediol via facile in-house synthesis. In this study, we prepared IPDI microcapsules using urea formaldehyde by in situ polymerization. Scanning micro-reference electrode (SMRE) technique was used to characterize the self-healing ability of epoxy resin coatings functionalized with IPDI microcapsules on the surface of reinforcing steel Q235.

\section{Materials and Methods}

All chemicals were obtained from Sigma Aldrich. Water used in all experiments was produced by a Millipore Milli-Q Plus 185 purification system with a resistivity of higher than $18.3 \mathrm{M} \Omega \mathrm{cm}$. All metals used in the experiments were purchased from Shanghai BaoSteel. The purity of copper was at 
least $99.99 \%$. Alkyd varnish was purchased from Lehua Inc. China. Air dry alkyd varnish is consisted of solvent naphtha and alkyd resin. The drying process of the alkyd varnish includes the solvent evaporates, hydroperoxide formation, hydroperoxide decomposition into free radicals, and polymerisation. All chemicals were used as received.

The conductivity of seawater $(4.863 \mathrm{~S} / \mathrm{m})$ and $\mathrm{pH}(7.36)$ were measured using a YSI 6600 (YSI Inc). Plasma treatment tests were carried out using a Model EPPs 2000, Plasmart Inc. China. Fourier Transform Infrared Spectroscopy (FT-IR) tests were performed on a Thermo Scientific Nicolet iN10, Thermo Fisher Scientific Inc. The measurements of the potential distribution on the steel surface were recorded by Scanning micro-reference electrode (SMRE) technique using a model CSPM5500-XMU-BY Being Nano-Instruments (Beijing, China) device. Alternating current scanning electrochemical microscopy (AC-SECM) experiments were carried out using a scanning electrochemical microscope from Sensolytics (Bochum, Germany). A platinum ultramicroelectrode (UME) of $25 \mu \mathrm{m}$ diameter served as AC-SECM tips.

\section{Results and Discussion}

FT-IR spectra of the self-healing products in the scratched crevice showed peaks for the isocyanate group (-NCO) at $2263^{-1}$ and $1337 \mathrm{~cm}^{-1}$ in Fig. 1. New bands due to the formation of urea groups appeared at $1637 \mathrm{~cm}^{-1}\left(\mathrm{C}=\mathrm{O}\right.$ stretching in urethane), $1560 \mathrm{~cm}^{-1}(\mathrm{~N}-\mathrm{H}$ bending in urea) (Fig. 1c). FT-IR spectra of AVC shows peak at $1731 \mathrm{~cm}-1$ that also exist in the crevice in Fig. 1c. It identified the scratched crevice cured by IPDI microcapsules.

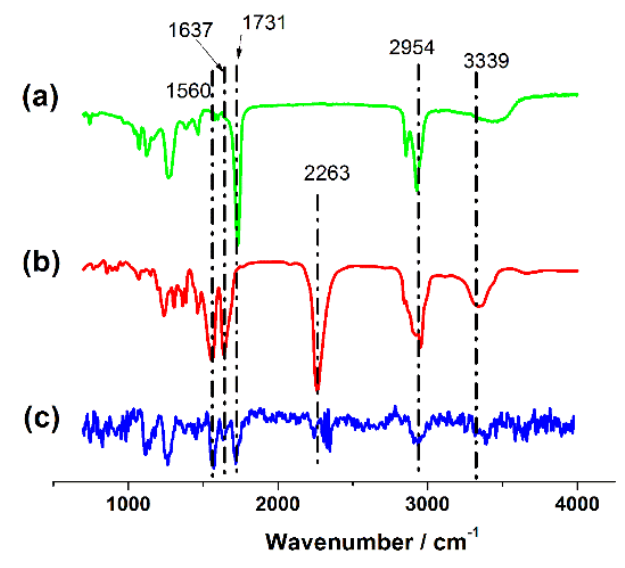

Fig. 1 FT-IR spectra of AVC (a), polyurethane (b), and self-healing products in the crevice (c), respectively.

Scanning micro-reference electrode (SMRE) technique was used to monitor the self-healing process of IPDI-AVC ( $20 \mu \mathrm{m}$ thickness $)$ by in situ imaging of the corrosion potential with good time and spatial resolutions. After scratching of the AVC surface, potential fields are measurable in the solution because of the exposure of the metal surface to water which allows electrochemical reactions between the metal and the seawater. A potential distribution map of the steel surface can be obtained by measuring potential differences between a scanning micro-probe and a micro-reference electrode (Fig. 2). The AVC coatings with IPDI microcapsules were scratched by a doctor blade. The width of the scratched crevice is about $30 \mu \mathrm{m}$. Then, the samples were immersed in $0.01 \mathrm{M} \mathrm{NaCl}$ solution. The potential distribution images over the surface of reinforcing steel in $\mathrm{NaCl}$ solution after immersion for different time are shown in Figure 2. There obviously existed a scratched line in the SMRE figure. The potential difference along the crevice shows the corrosion process which are shown in Figure 2-a, as reinforcing steel exposed in $\mathrm{NaCl}$ solution. Only several small peaks (less than $3 \mathrm{mV}$ ) were observed indicating that no localized corrosion occurred on the steel surface. After 2 hours, the potential difference changed less (Figure 2-b). Then, the IPDI liquid in microcapsules flow out of the core, and reacted with water after 4 hours. The coating shows the self-healing ability that partly cured the scratched crevice (Figure 2-c). For 8 hours immersion, the scratched crevice was well cured, and 
there was nearly no potential difference along the crevice line (Figure 2-d). Under this condition, the localized corrosion did not occur. The epoxy resin coatings functionalized by IPDI microcapsules has good self-healing ability.
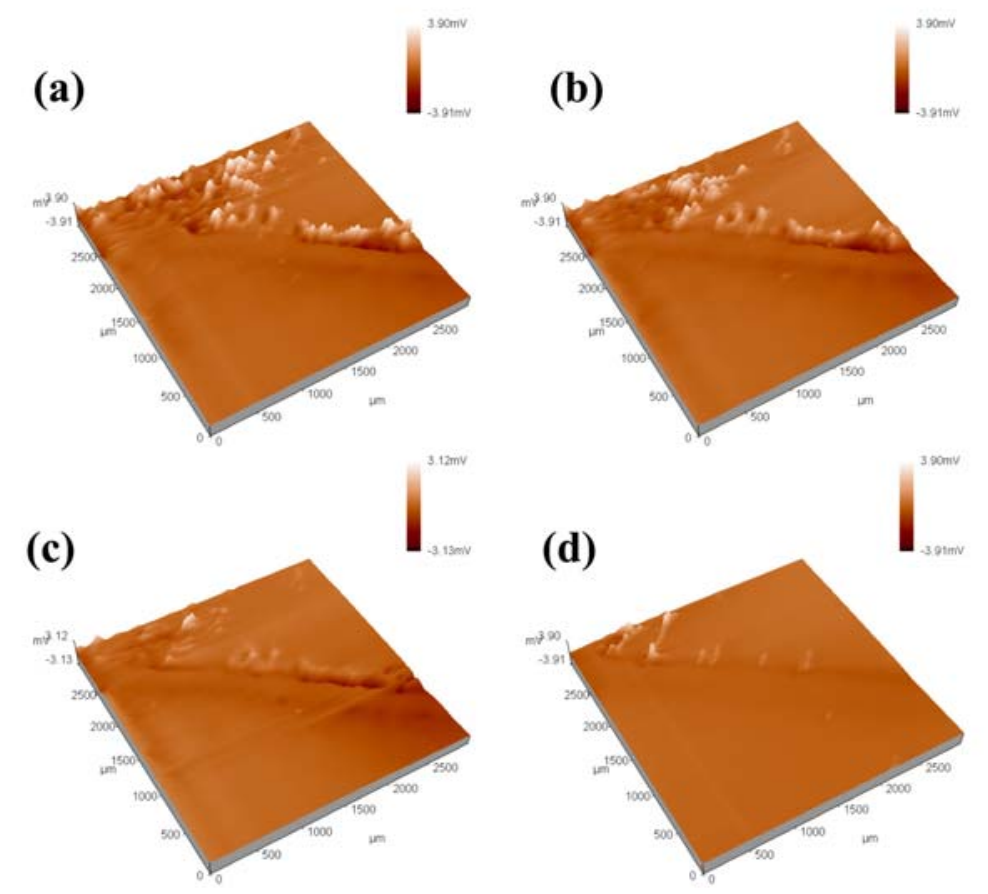

Fig. 2 Self-healing process of scratched smart coating on reinforcing steel surface for anticorrosion. The sample immersed in seawater: (a) $0.5 \mathrm{~h}$; (b) $2 \mathrm{~h}$; (c) $4 \mathrm{~h}$; (d) $8 \mathrm{~h}$.

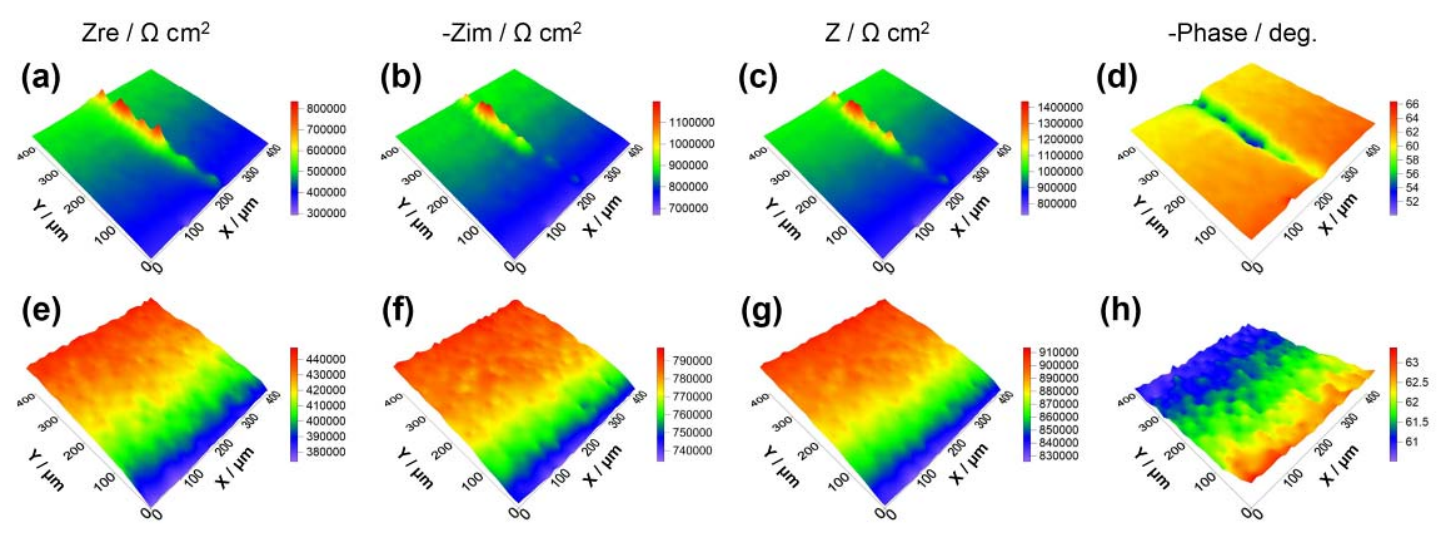

Fig.3 AC-SECM images of a self-healing coating surface immersed in $0.5 \mathrm{mM}$ ferrocene-methanol + $0.1 \mathrm{M} \mathrm{KCl}$ solution. DC potential: $0 \mathrm{~V}$ vs. $\mathrm{Ag} / \mathrm{AgCl} / \mathrm{KCl}(3 \mathrm{M})$. Amplitude of the AC potential signal: $10 \mathrm{mVpp}$, and frequency of $517 \mathrm{~Hz}$. Tip diameter: $25 \mu \mathrm{m}$; tip-substrate distance: $15 \mu \mathrm{m}$. (a-d) sample with crevice. (e-h) healed sample.

The choice of mediator was important for the SECM, as the solution should own the redox pair $\mathrm{Ox} / \mathrm{Red}$ as the necessity of monitoring feedback current. However, it was not necessary to measure the electrochemical signal by redox pair for AC-SECM test.

The 3D topography images of Zre, Zim, and $|\mathrm{Z}|$ at the frequency of $517 \mathrm{~Hz}$ could distinguish the area of the artificial defect in the self-healing coating that was approximately along the symmetry axis of the X direction of the sample surfaces. The form of "-phase" was used to express the distributions 
of the phase as it was an expression for the EIS test data. The graphs of "-phase" had the opposite direction of the impedance images.

The electrolyte exposed on the metal surface of the scratched crevice became electroactive due to the AC-perturbation influenced metal corrosion with dissolved oxygen in the AC-SECM test.

The images of Zre, Zim, and $|\mathrm{Z}|$ had the same tendency of showing the scratched crevice in Fig.3.

The healed sample surface still had the impedance differences for the 3D topography graphs of Zre, Zim, and $|Z|$. But the peak value of the impedance apparently decreased from $1447838 \Omega$ (Fig. 3c) to $913854 \Omega$ (Fig.3g).

The 3D "-phase" topography graphs of both the scratched crevice sample and healed sample had the same surface topography trend. But the scratched crevice was clearly observed for the scratched crevice sample. The difference value had a much more large range for the scratched crevice sample (Fig.3d) than that of the healed sample (Fig.3h).

\section{Summary}

In conclusion, a new self-healing coating was prepared by adding isocyanate microcapsules as functional additives to AVC. Optical images, SEM images and FT-IR spectra demonstrated the formation of the IPDI microcapsules. The self-healing ability of IPDI-AVC coating was investigated by SMRE and AC-SECM.

\section{Acknowledgement}

This paper is supported by National Natural Science Foundation of China (No. 51401185) and The Open Fund of Key Laboratory of Marine Environmental Corrosion and Bio-fouling, Institute of Oceanology, Chinese Academy of Sciences (No. MCKF201405).

\section{References}

[1] R. M. Latanision, Mater. Performance, 26, 9 (1987).

[2] K. Jud, H. H. Kausch, and J. G. Williams, J. Mater. Sci., 16(1981), p:204

[3] S. R. White, N. R. Sottos, P. H. Geubelle, J. S. Moore, M. R. Kessler, S. R. Sriram, et al. Nature, 409(2001), p:794

[4] D. Y. Wu, S. Meure, D. Solomon. Prog. Polym. Sci., 33 (2008), p: 479

[5] J. Yang, M. W. Keller, J. S. Moore, S. R. White and N. R. Sottos, Macromolecules, 41(2008), p: 9650

[6] W. Wang, L. Xu, F. Liu, X. Li and L. Xing, J. Mater. Chem. A, 1(2013), p: 776

[7] W. Wang, L. Xu, H. Sun, X. Li, S. Zhao and W. Zhang, J. Mater. Chem. A, 3(2015), p: 5599

[8] W. Wang, L. Xu, X. Li, Z. Lin,Y. Yang and E. An, J. Mater. Chem. A, 2(2014), p: 1914 